\title{
Distress in Cancer Patients and Their Caregivers and Association with the Caregivers' Perception of Dyadic Communication
}

\author{
Markus W. Haun ${ }^{a, b}$ Halina Sklenarova ${ }^{a}$ Anette Brechtel ${ }^{a, c}$ \\ Wolfgang Herzog ${ }^{a}$ Mechthild Hartmann ${ }^{a}$
}

aDepartment of General Internal Medicine and Psychosomatics, Heidelberg University Hospital, Germany; ${ }^{b}$ Soteria Bern, University Hospital of Psychiatry Berne, Switzerland; 'Division of Psycho-Oncology, National Center for Tumor Diseases, Heidelberg University Hospital, Germany

\section{Keywords}

Distress · Caregiver - Communication - Cancer - Anxiety · Disclosure

\section{Summary}

Background: Discrepancies within cancer-affected couples can disrupt security within the dyadic relationship during cancer treatment. This study investigated the patients' and caregivers' distress and associations between the caregivers' perception of the patients' degree of open communication and their distress. Participants and Methods: In a cross-sectional survey, 189 pairs of cancer patients (31\% gastrointestinal, 34\% lung, 35\% urological cancers) and their partners were assessed for distress (QSC-R10), depression and anxiety (PHQ-2/GAD-2). The caregivers also reported their perception of the patients' degree of disclosure regarding cancer-relevant topics (CCAT-F Disclosure subscale), caregiver strain (CSI), and unmet needs (SCNS-P\&C). Prevalences of clinically significant distress were calculated. Associations were calculated between the caregivers' and the patients' ratings and between the caregivers' distress and their perception of the patients' degree of disclosure. Results: $33 \%$ of the caregivers and $25 \%$ of the patients exhibited significant anxiety, with a tendency towards a higher frequency in the caregivers $(p=0.10)$. The prevalence of depression was lower but equally high in caregivers and patients. The caregivers' perceived non-disclosure by the patients was primarily associated with their anxiety $(r=0.31)$, disease-specific distress $(r=0.32)$, and psychological/emotional needs $(r=0.35)$. Conclusion: The identification of caregivers reporting problems in communicating with patients should be pursued in clinical practice as this might indicate that caregivers are particularly burdened.

Markus W. Haun and Halina Sklenarova contributed equally to this article.

\section{KARGER}

Fax +497614520714

Information@Karger.com

www.karger.com
(C) 2014 S. Karger GmbH, Freiburg

$2296-5270 / 14 / 0378-0384 \$ 39.50 / 0$

Accessible online at:

www.karger.com/ort

\section{Introduction}

The diagnosis of cancer not only affects the patients but also other people from their social background [1]. Caring for a patient with cancer usually does not end with the acute phase of the illness but continues after active treatment has ended [2]. In many cases, providing support and caring for somebody having cancer continues for several years. Sometimes it becomes a full-time job [3] including assistance in daily living activities or helping the ill person to manage different tasks [4]. This long-term care is very often carried out at home by significant others, like partners, parents, siblings, or children [5]. Therefore, cancer can be considered a family illness causing 'fears, uncertainty, disrupted life plans, rearranged schedules and routines, changed interpersonal communication, existential worries, alterations in household members' functioning, and heightened household tension' [6, 7]. Nijboer et al. [1] described caregiving as a dynamic process with several trajectories in which the caregivers' emotional well-being is at stake and a notable number of them are affected by significant anxiety and depression due to the uncertainty inherent to the disease [8]. There is also evidence for discrepancies between the patients and their caregivers regarding emotional and psychological strain. In a recently published meta-analysis, Mitchell et al. [9] reported that anxiety is more common than depression in caregivers and that the prevalence of anxiety in caregivers exceeds the prevalence in patients. Based on previous work indicating different experiences of the disease within the family regarding, e.g., coping strategies [10], symptoms causing distress and occurrence of the symptoms [11], or perception of pain [12], cancer-related communication constitutes an essential part of managing a cancer disease. In a study of 40 lung cancer patients and their caregivers, Zhang and Siminoff [13] found that $65 \%$ of the couples experienced communication problems. In line with this finding, one of our own studies investigating unmet needs of caregivers across different domains 
recently revealed unmet needs in the domain of communication and family [14]. The aim of this cross-sectional study was to investigate possible associations between low self-disclosure of cancer patients regarding disease-related topics and the emotional well-being of their caregivers.

\section{Methods}

\section{Study Design and Sample}

A cross-sectional survey of consecutively recruited pairs of cancer patients and their caregivers was conducted. The patients were either outor inpatients at the National Center for Tumor Diseases (NCT), Heidelberg University Hospital in Germany. The study was approved by the Heidelberg Medical School ethical commission.

\section{Eligibility and Recruitment}

The inclusion criteria allowed for adult patients with a definitive cancer diagnosis of gastrointestinal, urological, or lung tumor localization and required that both patient and caregiver agreed to participate in the study. Exclusion criteria for patients and caregivers were insufficient knowledge of the German language, cognitive impairment, and an unclear information status about the cancer diagnosis and stage. All patients of the gastrointestinal, urological, or lung cancer unit were contacted consecutively and invited to take part in the study, regardless of whether they were inpatients or outpatients. The caregivers were nominated by the patients and invited separately to take part in the study. Caregivers who did not accompany the patients were also invited; in this case, the questionnaire set was sent with the patient to be filled in at home. After agreement of the partner but prior to enrolment, informed consent was obtained from the participants. Patients and partners separately completed paper-pencil self-report questionnaires and returned them either directly in the hospital or from at home via separate sealed postage-paid envelopes. The participants were reminded via phone up to 2 times. Recruitment and data collection was undertaken in July/August 2012.

\section{Measurements}

Sociodemographic data were provided by the participants whereas medical data were obtained from the medical records and confirmed by the attending physician. Medical data such as tumor localization, occurrence and type of metastases, disease duration and stage were recorded according to the Basic Documentation for Psycho-Oncology (PO-Bado, [15]). For subsequent refusal/non-responder analysis, age, gender, and reason for refusal/non-response were documented. For all participating subjects, the questionnaire set comprised the Questionnaire on Distress in Cancer Patients - Short Form (QSC-R10) with a cut-off point of 14 [16] and the ultra-brief Patient Health Questionnaire-4 (PHQ-4) [17], the composite measure of the PHQ-2 and the Generalized Anxiety Disorder Scale-2 (GAD-2). The PHQ-2 is the short version of the 9-item PHQ-9 and evaluates core criteria for depression, depressed mood, and anhedonia [18]. GAD-2 represents a 2-item screener for generalized anxiety derived from the 7-item GAD-7 [19]. For clinical practice, a cut-off point of 3 or greater in the PHQ-2 or GAD-2 suggests clinically significant depression or anxiety, respectively.

Finally, only caregivers completed the Caregiver Strain Index (CSI) [20], the Supportive Care Needs Survey (SCNS-P\&C-G) [21, 22] for measuring supportive care needs across the information, healthcare services, daily living, and psychological domains, and the caregivers' version of the Disclosure Scale of the Cancer Communication Assessment Tool (CCAT-F) [23, 24]. This scale assesses the caregivers' perception of the patients' degree of open communication regarding cancer-related aspects (e.g. 'My family member does not tell me about his/her problems, because he/she things there is nothing I can do to help.' or 'My family member hesitates to mention treatment side effects to doctors and nurses.'). The cut-off on the disclosure scale of the CCAT-F was set at 3 , indicating that caregivers' ratings exceeding 3 indicate perceived non-disclosure in patients' communication about cancer. In addition to the above-mentioned instruments, 2 stand-alone items concerning the subjective need for psychological support and acceptance of support were included. Caregivers were asked if they 'have/don't have a need to seek psychological support' and 'would/would not accept psychological support'.

\section{Statistical Analysis}

Only pairs of questionnaire sets completed by both patients and caregivers were subjected to analysis undertaken with SAS $9.3^{\circledR} .4$ sets, in which $>40 \%$ of CCAT-F items were missing, were excluded from analysis. Descriptive statistics were calculated for sample characteristics, after distributions of variables were examined for skewness and outliers. To investigate the representativeness of the sample, a non-responder analysis was conducted, running Fisher's exact test for gender and tumor type and a $\mathrm{t}$-test for age.

The central aim of the study comprised, on the one hand, the comparison of prevalences of clinically significant distress between patients and caregivers. For this reason, Fisher's exact test was computed. On the other hand, we aimed at estimating associations between the caregivers' perception of the patients' degree of open communication regarding the disease and the caregivers' distress. Therefore, we calculated Pearson productmoment correlation coefficients between the CCAT-F total score and the caregivers' total scores of the GAD-2, PHQ-2, QSC-R10, and domains of unmet needs as operationalized in the SCNS-P\&C-G. For exploratory analysis of the sources of the caregivers' distress, we additionally calculated Pearson product-moment correlation coefficients between the CCAT-F total score and the caregivers' ratings on single items of the QSC-R10 and CSI. Due to the exploratory intention of this secondary analysis, no adjustments for multiple comparisons were made. For all tests, statistical significance was evaluated at a type 1 error of $5 \%$ (two-tailed).

\section{Results}

\section{Sample and Non-Responder Analysis}

Of the 331 screened patients, 267 fulfilled the inclusion criteria. Of these, 44 declined participation, 25 did not reply, and in 5 cases the questionnaires were only returned by the patient but not by the respective caregiver. Consequently, the final response rate was $72.3 \%$. The questionnaire sets of 4 pairs were excluded due to frequent missing caregiver data. Thus, complete questionnaires were obtained from 189 patientcaregiver pairs. All analyses were conducted for this subsample. Subjects who passively or actively refused participation did not differ significantly from those included with respect to age $(\mathrm{p}=0.60)$ and gender $(\mathrm{p}=0.66)$. However, lung cancer patients were more likely to refuse participation than patients suffering from urological or gastrointestinal tumors $(\mathrm{p}<0.001)$.

\section{Participant Characteristics}

The demographic and medical characteristics of the patients and their caregivers are given in table 1. The tumor entities were balanced with nearly equal parts: $35 \%$ of the patients were diagnosed with a urological tumor, $31 \%$ with a gastrointestinal tumor, and $34 \%$ of the patients suffered from lung cancer. The majority of the participating caregivers were partners to the cancer patient $(84 \%)$, followed by adult children $(12 \%)$. 


\section{Prevalence of Clinically Significant Depression,}

\section{Anxiety, and Cancer-Related Distress}

The prevalence of clinically significant mental burden is given in table 2 . Although there were no statistically significant differences between patients and caregivers regarding depression, anxiety, and disease-related distress, the analysis yielded a tendency towards a higher frequency of clinically significant anxiety in the caregivers $(p=0.10)$. Fisher's exact

Table 1. Demographic and medical characteristics of the patients and their caregivers

\begin{tabular}{lcc}
\hline & Caregivers, & Patients, \\
& $\mathrm{N}=189$ & $\mathrm{~N}=189$ \\
\hline Female & $138(73 \%)$ & $60(32 \%)$ \\
Age, years (mean \pm SD) & $58.2 \pm 12.4$ & $62.8 \pm 10.3$ \\
Education $\geq 9$ years & $108(57 \%)$ & $106(56 \%)$ \\
Employed & $75(40 \%)$ & $17(9 \%)$ \\
Tumor type & \\
Lung & $65(34 \%)$ \\
Kidney & $27(14 \%)$ \\
Prostatic & $19(10 \%)$ \\
Colon & $17(9 \%)$ \\
Rectum & $15(8 \%)$ \\
Pancreatic & $13(7 \%)$ \\
Bladder & $12(6 \%)$ \\
Other & $21(11 \%)$ \\
Time since diagnosis (median), weeks & 62.4 \\
Disease stage at assessment & $32(17 \%)$ \\
I-III & $142(75 \%)$ \\
IV & $15(8 \%)$ \\
Unknown & $16(8 \%)$ \\
Brain metastases & \\
RECIST [32] & $53(28 \%)$ \\
Progressive disease & $69(37 \%)$ \\
Stable disease & $20(11 \%)$ \\
Partial remission & $14(8 \%)$ \\
Complete response & $30(16 \%)$ \\
Unknown & \\
WHO/ECOG performance status ${ }^{\mathrm{a}}$ & \\
0 & \\
I & \\
II & \\
III & \\
IV & \\
\hline SD = Standard deviation, RECIST = Response Evaluation & \\
Criteria in Solid Tumors, WHO = World Health Organization & \\
ECOG = Eastern Cooperative Oncology Group. & \\
a 0 = Asymptomatic; I = symptomatic but completely ambulatory; & \\
II = symptomatic, < 50\% in bed during the day; III = symptomatic, \\
$>$ 50\% in bed, but not bedbound; IV = bedbound. \\
\hline
\end{tabular}

test for caregiver gender and clinically significant anxiety in the GAD-2 as well as depression in PHQ-2 and distress in QSC-R10 yielded no significant differences, indicating that male and female caregivers exhibited comparable prevalences of clinically relevant distress. Furthermore, we did not find any associations of the caregivers' distress neither with the patients' medical functional status or disease stage nor with the type of dyadic relationship (partner of the patient versus other family member). Furthermore, $23 \%$ of the caregivers reported unmet psychological and emotional needs, and an even higher proportion of all caregivers of $48 \%$ agreed to be willing to accept psychosocial support when offered.

\section{Associations between Caregivers' Perception of Patients' \\ Degree of Open Communication and Their Distress/ \\ Unmet Needs}

Overall, $13 \%$ of the caregivers reported perceiving low disclosure in the patients' communication about their cancer. There was no significant difference between male and female caregivers regarding the frequency of perceived low disclosure in the patients' communication. Moreover, we did not detect significant associations with disease characteristics (disease stage, functional impairment) or type of dyadic relationship (partner of the patient versus other family member).

The correlations between the caregivers' perception of the patients' degree of open communication and their distress as well as their unmet needs are given in table 3. The caregivers' perception of lower disclosure went along not only with higher levels of anxiety and disease-specific distress in the caregivers but also with more unmet psychological needs and needs in family communication. The association between the caregivers' perceived low degree of disclosure by the patients and depression in the caregivers turned out to be rather weak.

\section{Associations between Caregivers' Perception of Patients' Degree of Open Communication and \\ Components of Cancer-Related Distress in Caregivers}

The 5 strongest significant associations between the caregivers' perceived level of disclosure by the patients and components of cancer-related distress in the caregivers were

Table 2. Prevalence of clinically significant depression, anxiety, and cancer-related distress

\begin{tabular}{lrr}
\hline & Caregivers & Patients \\
\hline Anxiety (GAD-2) & & \\
$\quad$ Within normal limits & $121(64 \%)$ & $132(73 \%)$ \\
$\quad$ Elevated & $67(36 \%)$ & $50(27 \%)$ \\
Depression (PHQ-2) & $133(73 \%)$ & 0.10 \\
$\quad$ Within normal limits & $50(27 \%)$ & $132(71 \%)$ \\
$\quad$ Elevated & $89(48 \%)$ & 0.90 \\
Cancer-related distress (QSC-R10) & $98 \%)$ \\
$\quad$ Within normal limits & $98(52 \%)$ & $108(58 \%)$ \\
Elevated & 0.35 & \\
\hline
\end{tabular}

GAD-2 = Generalized Anxiety Disorder Scale-2, PHQ-2 = Patient Health Questionnaire-2; QSC-R10 = Questionnaire on Distress in Cancer Patients-Short Form.

*p value for comparison of patients and caregivers regarding the prevalence of clinically significant distress (Fisher's exact test). 
Table 3. Pearson correlation coefficients for the caregivers' perception of the patients' degree of open communication, with total scores of distress instruments

\begin{tabular}{ll}
\hline & $\begin{array}{l}\text { Correlation with caregivers' perceived degree of disclosure by the patients } \\
\text { (CCAT-F) }\end{array}$ \\
\hline Anxiety (GAD-2) & $\mathrm{r}=0.31^{* *}$ \\
Depression (PHQ-2) & $\mathrm{r}=0.20^{*}$ \\
Significant cancer-related distress (QSC-R10) & $\mathrm{r}=0.32^{* *}$ \\
CSI & $\mathrm{r}=0.28^{* *}$ \\
Psychological and emotional needs (SCNS-P\&C-G) & $\mathrm{r}=0.35^{* *}$ \\
Family and communication needs (SCNS-P\&C-G) & $\mathrm{r}=0.26^{*}$ \\
\hline
\end{tabular}

CCAT-F = Disclosure Scale of the Cancer Communication Assessment Tool, GAD-2 = Generalized Anxiety Disorder Scale-2,

PHQ-2 = Patient Health Questionnaire-2, QSC-R10 = Questionnaire on Distress in Cancer Patients - Short Form, CSI = Caregiver Strain Index, SCNS-P\&C-G = Supportive Care Needs Survey, patients and caregivers - German version.

$* \mathrm{p}<0.01, * * \mathrm{p}<0.0001$.

Table 4. Pearson correlation coefficients for the caregivers' perception of the patients' degree of open communication with single items from instruments measuring cancer-related distress in caregivers

\begin{tabular}{|c|c|c|c|}
\hline Instrument & Item no. & Item wording & $\begin{array}{l}\text { Correlation with caregivers' perceived degree } \\
\text { of disclosure by the patients (CCAT-F) }\end{array}$ \\
\hline QCS-R10 & 6 & It is difficult for my partner to empathize with my situation. & $\mathrm{r}=0.41 * *$ \\
\hline CSI & 9 & Some behavior is upsetting. & $\mathrm{r}=0.33 * *$ \\
\hline CSI & 13 & $\begin{array}{l}\text { I feel completely overwhelmed (e.g. because of worry about } \\
\text { concerns about how I will manage). }\end{array}$ & $\mathrm{r}=0.30 * *$ \\
\hline QSC-R10 & 10 & I feel tense and/or nervous. & $r=0.26^{*}$ \\
\hline CSI & 3 & $\begin{array}{l}\text { It is a physical strain (e.g. because of lifting in and out of a chair; } \\
\text { effort or concentration is required). }\end{array}$ & $\mathrm{r}=0.25^{*}$ \\
\hline
\end{tabular}

CCAT-F $=$ Disclosure Scale of the Cancer Communication Assessment Tool,

QSC-R10 = Questionnaire on Distress in Cancer Patients - Short Form, CSI = Caregiver Strain Index.

$* \mathrm{p}<0.001, * * \mathrm{p}<0.0001$.

revealed on the item level of the QSC-R10, and the CSIs are presented in table 4 . The total number of analyzed items from both questionnaires was 23; hereof, 16 were significantly correlated with perceived disclosure (CCAT-F).

\section{Discussion}

This study investigated (1) the prevalence of depression, anxiety, and cancer-specific distress in patients with heterogeneous tumor diagnoses and in their primary caregivers and (2) associations between the caregivers' perception of the patients' degree of open communication and the levels of the caregivers' distress. Methodological limitations regarding the generalizability of the presented results lie in the crosssectional study design impeding causal inferences from bivariate correlations between disclosure and distress scores. The detected prevalences of depression and anxiety were comparable to previous findings. In a recent meta-analysis, Mitchell et al. [9] found a prevalence of depression of $26 \%$ in patients versus $26 \%$ in their spouses and a prevalence of anxiety of $28 \%$ in patients versus $40 \%$ in their spouses. Thus, the caregivers' tendency to more frequently exhibit significant anxiety compared to the patients was confirmed. Nevertheless, prior studies showed that, within couples coping with cancer, women tend to report more distress than men, regardless of their roles (patient/caregiver) [25]. However, the analysis in our study revealed no significant difference between female and male caregivers regarding prevalence of anxiety.

Associations between non-disclosure of cancer-related topics and caregivers' disease-related distress have been empirically investigated only in a few previous studies [24, 26-29]. The correlations with caregiver strain yielded in our study were somewhat higher $(r=0.28$ vs. 0.12$)$ than in an earlier one [30] conducted in a homogeneous sample of gastrointestinal cancer patients and their spouses. This could be a possible effect of the higher frequency of patients with very advanced disease in our population. However, the abovepresented associations between the caregivers' perceived degree of disclosure by the patients and the caregivers' levels of distress were comparable to data (Hospital Anxiety and Depression Scale (HADS); $\mathrm{r}=0.44$ with $\mathrm{p}<0.0001$ ) published by Kornblith et al. [27] some years ago who applied the Cancer-Related Communication Problems within Couples Scale (CRCP) in a sample of lung cancer patients. Regarding depression, the identified correlations are in line with those detected in the original validation study of the CCAT-PF (Center for Epidemiological Studies Depression Scale $(\mathrm{CES}-\mathrm{D}) ; \mathrm{r}=0.18$ with $\mathrm{p}<0.01)$ [17], which also demonstrated associations between conflict on the caregivers' side as operationalized in the Family Environment Scale (FES) and the caregivers' perceived lower communication in patients. This tendency of absent communication about cancer-related topics perceived by the caregivers going along with conflict 
could be further clarified by considering single-item correlations regarding cancer-specific burden in our study. Caregivers who perceived that patients avoided talking about the disease and its implications reported also a lack of empathy from the side of the patient as well as worries about managing the disease trajectory. Besides fear of progression, this perception is further connected to unmet psychological and emotional needs.

\section{Conclusions}

In accordance with previous findings, our results underline the high prevalence of particularly anxiety in caregivers: 1 in 3 caregivers in our study exhibited clinically significant anxiety as operationalized in the GAD-2. Regarding the caregivers' perceived degree of open communication of the patient on cancer-related topics, 1 in 6 caregivers observed low levels of disclosure by the patients. This perception was associated with high levels of anxiety and distress in the caregivers and higher unmet needs in the psychological and emotional domain. The sources of the caregivers' distress related to the perception of restricted communication mainly comprised their perception of the patients' lack of empathy and concerns about managing the disease in the future. Thus, these different components point to the complexity of possible relationships between the caregivers' perceived low level of the patients' disclosure and their distress. Consequently, the identification of caregivers reporting problems in communicating with the patients should be pursued in clinical practice as this might indicate that caregivers are particularly burdened. With regard to previous findings that the mere disclosure of emotions and thoughts to one's intimate partner might not always be beneficial in reducing distress [26], further assessment of the communication patterns may be necessary. This can be a wellchosen time point to consider professional psycho-oncological services for caregivers of cancer patients [31].

\section{Disclosure Statement}

All authors declare that there is no conflict of interest.

\section{References}

$\checkmark 1$ Nijboer C et al.: Cancer and caregiving: the impact on the caregiver's health. Psychooncology 1998; 7:3-13.

2 Given BA, Sherwood P, Given CW: Support for caregivers of cancer patients: transition after active treatment. Cancer Epidemiol Biomarkers Prev 2011;20:2015-2021.

3 Kim Y, Schulz R: Family caregivers' strains: comparative analysis of cancer caregiving with dementia, diabetes, and frail elderly caregiving. J Aging Health 2008;20:483-503.

4 Girgis A, Lambert S: Caregivers of cancer survivors: the state of the field. Cancer Forum 2009; $33: 168$

5 Nijboer $\mathrm{C}$ et al.: Patterns of caregiver experiences among partners of cancer patients. Gerontologist 2000;40:738-746.

6 Lewis FM: The family's 'stuck points' in adjusting to cancer; in Holland JC, Breitbart WS, Jacobsen PB (eds): Psycho-Oncology. New York, Oxford University Press, 2009, pp 511-515.

7 Manne SL et al.: Spousal negative responses to cancer patients: the role of social restriction, spouse mood, and relationship satisfaction. J Consult Clin Psychol 1999;67:352-361.

8 Grov EK et al.: Anxiety, depression, and quality of life in caregivers of patients with cancer in late palliative phase. Ann Oncol 2005;16:1185-1191.

$\checkmark 9$ Mitchell AJ et al.: Depression and anxiety in longterm cancer survivors compared with spouses and healthy controls: a systematic review and metaanalysis. Lancet Oncol 2013;14:721-732.

10 Kershaw T et al.: Coping strategies and quality of life in women with advanced breast cancer and their family caregivers. Psychol Health 2004;19: 139-155.

11 Broberger E, Tishelman C, von Essen L: Discrepancies and similarities in how patients with lung cancer and their professional and family caregivers assess symptom occurrence and symptom distress. J Pain Symptom Manage 2005;29:572-583.
12 Miaskowski C et al.: Differences in patients' and family caregivers' perceptions of the pain experience influence patient and caregiver outcomes. Pain 1997;72:217-226.

13 Zhang AY, Siminoff LA: Silence and cancer: why do families and patients fail to communicate? Health Commun 2003;15:415-429.

14 Sklenarova $\mathrm{H}$ et al.: Psychometric evaluation of the German version of the Supportive Care Needs Survey for Partners and Caregivers (SCNS$\mathrm{P} \& \mathrm{C}-\mathrm{G}$ ) of cancer patients. Eur J Cancer Care, submitted.

15 Herschbach P et al.: The Basic Documentation for Psycho-Oncology (PO-Bado) - an expert rating scale for the psychosocial experience of cancer patients. Onkologie 2008;31:591-596.

16 Book $\mathrm{K}$ et al.: Distress screening in oncology evaluation of the Questionnaire on Distress in Cancer Patients - Short Form (QSC-R10) in a German sample. Psychooncology 2011;20:287-293.

17 Kroenke K et al.: An ultra-brief screening scale for anxiety and depression: the PHQ-4. Psychosomatics 2009;50:613-621.

18 Kroenke K, Spitzer RL, Williams JBW: The Patient Health Questionnaire-2: Validity of a two-item depression screener. Med Care 2003;41:1284-1292.

19 Kroenke K et al.: Anxiety disorders in primary care: prevalence, impairment, comorbidity, and detection. Ann Intern Med 2007;146:317-325.

20 Robinson BC: Validation of a caregiver strain index. J Gerontol 1983:38:344-348

21 Girgis A, Lambert S, Lecathelinais C: The supportive care needs survey for partners and caregivers of cancer survivors: development and psychometric evaluation. Psychooncology 2011;20:387393.

22 Lehmann C, Koch U, Mehnert A: Psychometric properties of the German version of the ShortForm Supportive Care Needs Survey Questionnaire (SCNS-SF34-G). Support Care Cancer 2012; 20:2415-2424.
23 Haun MW et al.: Assessing patient-caregiver communication in cancer - a psychometric validation of the Cancer Communication Assessment Tool (CCAT-PF) in a German sample. Support Care Cancer 2014;Apr 11 [Epub ahead of print].

24 Siminoff LA et al.: The Cancer Communication Assessment Tool for Patients and Families (CCAT-PF): a new measure. Psychooncology 2008;17:1216-1224.

25 Hagedoorn M et al.: Distress in couples coping with cancer: a meta-analysis and critical review of role and gender effects. Psychol Bull 2008;134:1-30.

26 Hagedoorn $\mathrm{M}$ et al.: Is self-disclosure in couples coping with cancer associated with improvement in depressive symptoms? Health Psychol 2011; 30:753-762.

27 Kornblith $\mathrm{AB}$ et al.: Cancer-related communication between female patients and male partners scale: a pilot study. Psychooncology 2006;15:780794.

28 Manne S et al.: Couples' support-related communication, psychological distress, and relationship satisfaction among women with early stage breast cancer. J Consult Clin Psychol 2004;72:660-670.

29 Song L et al.: Study of dyadic communication in couples managing prostate cancer: a longitudinal perspective. Psychooncology 2012;21:72-81.

30 Porter LS et al.: Disclosure between patients with gastrointestinal cancer and their spouses. Psychooncology 2005;14:1030-1042.

31 Northouse LL et al.: Interventions with family caregivers of cancer patients: meta-analysis of randomized trials. CA Cancer J Clin 2010;60:317339.

32 Therasse P et al.: New guidelines to evaluate the response to treatment in solid tumors. European Organization for Research and Treatment of Cancer, National Cancer Institute of the United States, National Cancer Institute of Canada. J Natl Cancer Inst 2000;92:205-216. 